\title{
Maxwell Equations on Unstructured Grids Using Finite-Integration Methods
}

\author{
W.J. Schoenmaker*, P. Meuris*, E. Janssens*, \\ K.-J. van der Kolk ${ }^{\dagger}$, N. van der Meijs ${ }^{\dagger}$ and W.H.A. Schilders ${ }^{+}$ \\ *MAGWEL, B-3001 Leuven, Belgium \\ $\{$ wim|peter|erik\}@magwel.com \\ $\dagger$ Delft University of Technology, 2600 AA Delft, Netherlands \\ $\{$ keesjan|nick\}@cobalt.et.tudelft.nl \\ ${ }^{+}$NXP, 5656 AE Eindhoven, Netherlands \\ wil.schilders@nxp.com
}

\begin{abstract}
It is announced that the Maxwell equations can be solved on unstructured grids using finite integration methods. Numerical experiments show that next to the finite-volume method, a discretization technique can be defined based on 'finite-surface' integration.
\end{abstract}

\section{Introduction}

Electromagnetic field simulations are extensively used for characterizing interconnects and integrated passives at high frequencies. Apart from the approach based on subdividing the field problem into 'partial equivalent electric circuits' (PEEC), the most common approaches are based on generalized finite-element methods (FEM) or finiteintegration technique (FIT). An in-depth view informs us that there is an important distinction between FEM and FIT: whereas FEM approximates a continuous function over the simulation domain by a piecewise continuous function [1, 2], FIT does not provide knowledge of the function between the grid points. The finite-integration method considers the values at the grid points as 'fair' representatives of the values in the surrounding of the grid points, no further assumptions are made what the precise values must be at locations where no grid point is placed. A consequence of the viewpoint of FIT is that discretized equations are derived from balance equations, where fluxes from one volume element into neighboring ones must satisfy conservation laws. In other words: solving for example the differential equation

$$
\nabla \cdot \mathbf{J}+\frac{\partial \rho}{\partial t}=0
$$

requires that the following algebraic differential equations are solved:

$$
\sum_{j=1}^{n_{i}} J_{i j} \Delta S_{i j}+\frac{\partial \rho_{i}}{\partial t} \Delta v_{i}=0 \forall i
$$


where $J_{i j}$ is a discretized version of the current density between node $i$ and node $j$ and $\rho_{i} \Delta v_{i}$ is the nodal value of the charge density weighted with the nodal volume. In particular, if $\mathbf{J}=-\sigma \nabla V$ then a good representative for this current is $J_{i j}=-\sigma\left(V_{j}-\right.$ $\left.V_{i}\right) / h_{i j}$, where $h_{i j}$ is the distance between node $i$ and node $j$. The finite-integration method is a well-known and widely used method in TCAD, since scalar quantities such as $p$ and $n$ as well as $V$ (the electrostatic potential) fit nicely into above view point. FIT is also known as the finite-volume method (FVM).

\section{The Maxwell-Ampere equation}

Recently, we have extended the finite-integration method to include vector variables $[3,4]$. In particular, the vector potential $\mathbf{A}$ that describes the magnetic induction, $\mathbf{B}=$ $\nabla \times \mathbf{A}$ is placed on the computational grid starting from Ampere's law

$$
\nabla \times\left(\frac{1}{\mu} \nabla \times \mathbf{A}\right)=\mathbf{J}+\frac{\partial \mathbf{D}}{\partial t} .
$$

By considering a link, $l$ in the computational grid and its associated dual surface $\Delta S_{l}$, we find that the discretized version of (3) becomes

$$
\sum_{l k} \Lambda_{l k} A_{k} / \mu_{k}=J_{l} \Delta S_{l}+\frac{\partial D_{l}}{\partial t} \Delta S_{l}
$$

where $\Lambda$ are geometrical factors. Whereas the transition in going from (1) to (2) required the use of Gauss' theorem, the use of Stokes' theorem is needed in obtaining (4) from (3).

It is an interesting fact to note that Gauss' theorem and Stokes' theorem are two realizations of the fundamental theorem of differential geometry

$$
\int_{\partial \Omega} \omega=\int_{\Omega} \mathrm{d} \omega
$$

which merely expresses the fact that the method of partial integration has a very general validity. In particular, Gauss' theorem corresponds to $\omega=\mathbf{D} \cdot \mathrm{d} \mathbf{S}$ and $\partial \Omega$ is the enclosing 2-D surface of a 3-D volume $\Omega$ whereas $\mathrm{d} \omega=\nabla \cdot \mathbf{D} \mathrm{d}^{3} v$. In a similar manner, Stokes' theorem is obtained by taking $\omega=\mathbf{A} \cdot \mathrm{d} \mathbf{l}, \partial \Omega$ is the enclosing 1-D contour of a 2-D surface $\Omega$ and $\mathrm{d} \omega=\nabla \times \mathbf{A} \cdot \mathrm{d}^{2} \mathbf{S}$. It should also be noted that the differential character of the vector needs to be respected when applying (5). That means that $\mathbf{D}$ is a two-form i.e. a vector that corresponds to a flux or current density and $\mathbf{A}$ must be a one-form i.e. a vector that corresponds to a velocity or field intensity).

Whereas the results in $[3,4]$ were obtained on structured grids we demonstrate that the Maxwell-Ampere equation can also be obtained on unstructured grids using a variant of the finite-integration method. In particular, the Stokes' theorem applies on general Delaunay grids. It should be emphasized that this is a highly non-trivial observation, since for obtuse volume elements links can have a negative dual area. The evaluation of the circulation around links with negative dual area is compensated by link contributions with large positive dual area such that the net result is in full agreement with the results obtained in grids which do not contain obtuse volume element. 
In fact, above observation leads to the conclusion that starting from (5), one must adapt the discretization scheme to the differential geometrical character of the equation that needs to be solved. The finite-volume method (FVM) naturally matches to discretize the balance equation as shown in (1). In an analogous way, a 'finite-surface method' (FSM) is suitable to discretize the equation (3).

\section{Demonstration}

We demonstrate the validity of our claim by computing the inductance of an integrated spiral inductor as shown in Fig. 1 (a), using different meshes. The first mesh has a
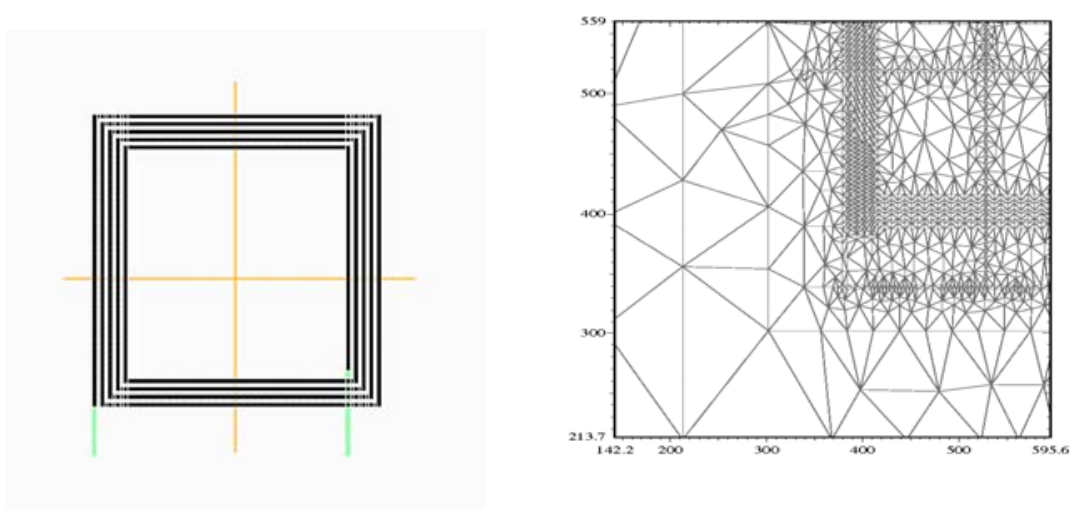

Figure 1: Layout of the spiral inductor (a). Zoom-in of an unstructured grid (b).

structured grid while the second grid contains generalized elements, but all elements are acute. The third grid also contains obtuse elements which is in general the result from a Delaunay algorithm. Fig. 1 (b) shows a detailed view on one of the unstructured grids that is used. In Fig. 2, the results for the inductance are shown using a structured grid as well as an unstructured grid. Excellent agreement is observed. The computation is achieved by solving the Maxwell-Ampere equation as well as the Poisson equation with voltage boundary conditions. After obtaining balance of the currents, the S-parameters are computed from a two-port system. Each port contains one contact at the inductor and one contact attached to the substrate. Finally, the inductance (and resistance) is extracted from the S-matrix.

\section{Conclusion}

We announce the possibility to discretize the Maxwell-Ampere system on unstructured grids such as 3D Delaunay grids, octree grids and variations thereof. All that is required is that the nodes of the volume cell are located on a sphere and that no other grid point is found in this sphere. The underlying discretization is a generalization of the finite-integration method. One does not need to refer to Nedelec's edge conformal base 


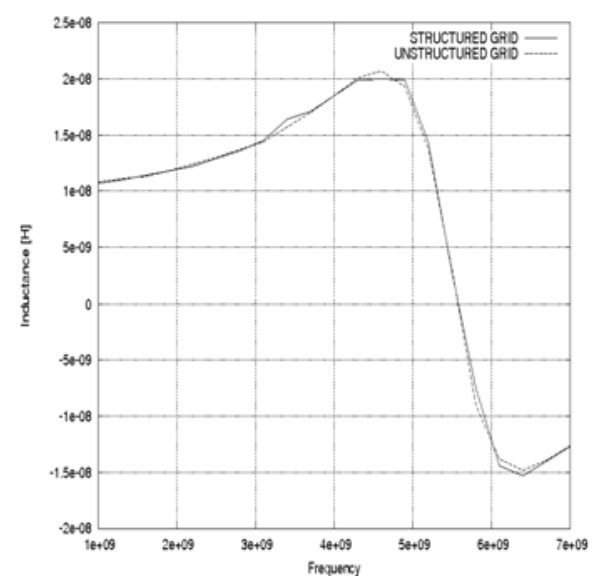

Figure 2: Inductances obtained on a structured and unstructured grid.

functions. However, one must assign degrees of freedom to the links of the computational grid as proposed in [3, 4]. The consequence of above observation is far reaching: existing device simulators can now be upgraded to solve the Maxwell-Ampere system. Although a mathematical proof is still lacking that the finite-surface method is a valid approach, numerous simulation experiments indicate that the method is sound. An important ingredient for the validity of the method is that the underlying grid must satisfy the Delaunay criterion. Unstructured grids can be created that do not obey the Delaunay criterion, yet leading to solutions that satisfy current balance, but the values of the currents do not converge to the correct limits for refined grids. Grids that do satisfy the Delaunay criterion do not suffer from this incorrect asymptotic behavior.

The present work was not motivated by the desire to perform computation on grids with many obtuse elements, but to address industrial applications. For the latter it is essential to compute on unstructured grids, since the complexity of such structures, would lead to far too-many grid nodes on structured grids.

\section{Acknowledgements}

We gratefully acknowledge financial support from the European Union for the projects CHAMELEON-RF, nanoCMOS and puIINANO.

\section{References}

[1] J.F. Lee, D.K. Sun and Z.J. Cendes, "Tangential vector finite elements for electromagnetic field computation", IEEE Trans. on Magnetics, vol. 27, 4032-4035, 1991.

[2] J.C. Nedelec, "Mixed Finite elements in $R^{3}$ ", Numer. Math. vol. 35, 315-341,1980.

[3] W. Schoenmaker, P. Meuris, and W. Magnus, "Ghost Fields in Classical Gauge Theories", Phys. Rev. Lett. vol. 88, 18162-1-18162-4, 2002.

[4] P. Meuris and W. Schoenmaker, "Strategy in electromagnetic interconnect modeling" IEEE Trans. on CAD. vol. 20, 739-748, 2001. 